\title{
Liquefaction of plant biomass for use in polymers - is it the right strategy?
}

\author{
J. Karger-Kocsis* \\ Polymer Technology, Faculty of Engineering and the Built Environment, Tshwane University of Technology, Pretoria 0001, \\ Republic of South Africa, and Department of Polymer Engineering, Faculty of Mechanical Engineering, Budapest \\ University of Technology and Economics, H-1111 Budapest, Hungary
}

Liquefaction is a widely practiced technology to convert gaseous and solid products into liquids. Biomass is considered as natural, renewable, combustible, high molecular weight material in which the energy of sunlight is stored - via photosynthesis - in the form of chemical bonds. From the viewpoint of chemical structure the plant biomass consists of cellulose, hemicelluloses and lignin which fall under the heading of lignocellulose. However, the term 'plant biomass' covers not only forestry products but also wastes from the agriculture, agroindustry and even municipal/domestic ones (e.g. waste paper). In the past great efforts were dedicated to the conversion of biomass into fuels. Recent developments target, however, is its conversion into raw materials for polymer synthesis and modification. This R\&D direction is driven by the facts that biomass is permanently regenerated, it is carbon neutral (i.e. no extra $\mathrm{CO}_{2}$ emission being the major cause of global warming), and it may compete with crude oil as suitable feedstock for polymer production. In order to break up the chemical backbone and functionalize the related 'fragments' various hydrolytic and solvolytic (solvents as phenol, di- and polyols, acetone etc.) techniques with and without additional catalysts (alkali or acidic types) are adapted. The resulting highly reactive 'black liquor' can be used as raw material to produce phenolic resins and polyurethanes due to its phenolic and alcoholic hydroxyl groups. It is, however, not an easy task to guarantee the quality of the decomposition products derived from a natural feedstock. This requires the development of an online process control of liquefaction. It can hardly be

\footnotetext{
${ }^{*}$ Corresponding author, e-mail: karger@pt.bme.hu (c) BME-PT
}

done without fundamental analytical studies on the liquefaction of suitable model compounds. Moreover, there is a need for value-added liquefied compounds allowing us their diversified use. Instead of costly separation afterwards, the liquefaction process should yield the target compound. The process is expected to be environmentally friendly and thus harmful solvents like phenol should be replaced by other ones. In this respect supercritical fluids and ionic liquids may have an important role. Possible value added products from the liquefaction of plant biomass are further thermosets, such as epoxy and unsaturated polyester resins. It would be of interest to check whether the partial decomposition of lignocellulosic materials (resulting in cellulose nanowhiskers) could be combined with an in situ polymerization molding to produce novel thermoset nanocomposites. It is obvious that the liquefied products are more costly raw materials for polymers than those from fossil feedstock. However, this may change, also by legislative actions, provided that the technical feasibility of thermochemical liquefaction of biomass and the straightforward use of the related products are confirmed. This is the task for the near future in order to give the right reply to the question posed in the title!

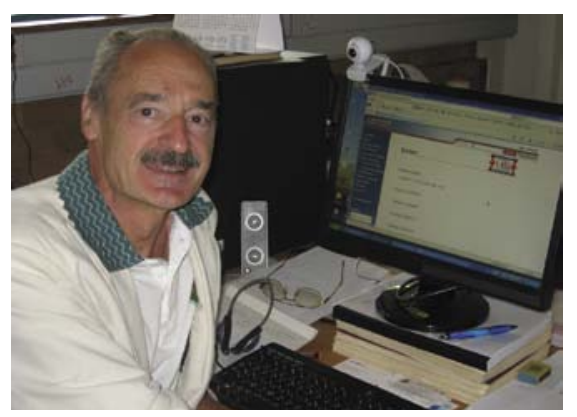

Prof. Dr.-Ing. Dr.hc. J. Karger-Kocsis, editor-in-chief 